\title{
Potential Impact of Populations Drift on Botrytis Occurrence and Resistance to Multi- and Single-Site Fungicides in Florida Southern Highbush Blueberry Fields
}

\author{
A. Amiri, ${ }^{\dagger}$ Department of Plant Pathology, Tree Fruit Research and Extension Center, Washington State University, Wenatchee 98801 ; and \\ A. I. Zuniga and N. A. Peres, Gulf Coast Research and Education Center, University of Florida, Wimauma 33598
}

\begin{abstract}
Incidence of blossom blight and Botrytis fruit rot (BFR), caused by Botrytis cinerea, on two southern highbush blueberry cultivars was evaluated in several blueberry fields grown in the vicinity $\left(\mathrm{BB}-\mathrm{Str}^{(+)}\right)$or not $\left(\mathrm{BB}-\mathrm{Str}^{(-)}\right)$of strawberry fields in central Florida. Blossom blight and BFR incidence were higher in BB-Str(+) fields in 2014 and significantly higher in 2015 compared to BB-Str ${ }^{(-)}$fields. In total, 613 B. cinerea isolates (i.e., 181 and 432 isolates from BB-Str ${ }^{(-)}$and BB-Str ${ }^{(+)}$fields, respectively) were collected. The isolates were evaluated for sensitivity to eight single-site and one multisite fungicides using a spore germination and a germ tube elongation assay. Overall, 5, 15, 24, 28, 54, and 93\% of isolates collected from BB-Str ${ }^{(-)}$were resistant to penthiopyrad, cyprodinil, boscalid, fenhexamid, pyraclostrobin, and thiophanate-methyl, respectively. Respective resistance frequencies in BB-Str ${ }^{(+)}$isolates were 10, 30, $65,66,89$, and $99 \%$. Resistance frequencies for all fungicides were

always higher in BB-Str ${ }^{(+)}$fields compared to BB-Str ${ }^{(-)}$fields. Isolates exhibiting resistance to six or five fungicides simultaneously were predominant (50 to 70\%) in blueberry fields regardless if they were grown in the vicinity of strawberry fields or not. Among 308 and 305 B. cinerea isolates tested in 2014 and 2015, 41.8 and $47.1 \%$, respectively, showed reduced sensitivity to the multisite fungicide captan. The lower label rate of captan applied preventively did not control isolates with reduced sensitivity on detached blueberry fruit. These findings suggest a potential population flow between strawberry and blueberry fields that may impact blossom blight and gray mold development in blueberry fields The relatively lower fungicide input applied to blueberry fields compared with strawberry fields seems to be sufficient to select for resistance and multiple-resistant phenotypes in $B$. cinerea populations in blueberry.
\end{abstract}

Blueberry production in Florida has increased steadily in the last 10 years, making the state among the top 10 producers in the United States (USDA-NASS 2016). Several low-chill blueberry cultivars have been selected, mainly for fresh market, in the last decade and grown between the $27^{\circ} \mathrm{N}$ and $30^{\circ} \mathrm{N}$ parallels. Southern highbush blueberry (SHB), which shares some climatic requirements with strawberry (Fragaria $\times$ asnanassa), is an interspecific hybrid of highbush blueberry (Vaccinium corymbosum), rabbiteye blueberry (V. virgatum), and Darrow's blueberry (V. darrowi), and is predominant in Florida, followed by rabbiteye blueberry, mainly grown in north Florida.

The flowering period of most SHB blueberry cultivars starts as early as February in Florida and continues until late April, whereas the first fruit are harvested in April. During this period, mild temperatures and extended wetness periods can be conducive to the devastating blossom blight caused by Botrytis cinerea (teleomorph Botryotinia fuckeliana (de Bary) Whetzel). In Florida, most blueberry fruit are shipped fresh (Williamson et al. 2015) and, under high disease pressure, Botrytis cinerea can cause severe damage to the fruit, especially to those intended for relatively longer postharvest storage periods. Other major diseases of blueberry fruit are anthracnose and mummy berry caused by Colletotrichum acutatum and Monilinia vaccinii-corymbosi, respectively (Batra 1983; Burchhardt and Cubeta 2015; Gillett and Schilder 2009). Interestingly, strawberry crops in Florida are grown annually between October and March, in the western-central part of the state (around the 28th parallel north). This region represents about $35 \%$ of the state blueberry production and is viewed as the area with a greater growth potential (Williamson et al. 2015). Blueberry fields are commonly grown next to strawberry fields in central Florida. The beginning of the flowering

${ }^{\dagger}$ Corresponding author: A. Amiri; E-mail: a.amiri@wsu.edu

Funding: Support for this study was provided by the Specialty Crop Block initiative fund under project number 2010-51181-21113.

Accepted for publication 23 April 2018.

() 2018 The American Phytopathological Society blueberry season in February overlaps with the peak production period in strawberry. During this period, high Botrytis pressure, due to the release of a considerable amount of inoculum in the atmosphere, is very common in this area of production.

Management of blossom blight and Botrytis fruit rot (BFR) is achieved through multiple fungicide applications. In SHB cultivars, phthalimide-captan (PHT) and the single-site fungicides fenhexamid (hydroxyanilide [Hyd]), pyraclostrobin (quinone outside inhibitor [QoI]), boscalid (succinate dehydrogenase inhibitor [SDHI]), cyprodinil (anilino-pyrimidine [AP]), and fludioxonil (phenylpyrrole [PP]) have been regularly used to manage blossom blight, BFR, and other major diseases in Florida blueberry fields. These fungicides are commonly used in strawberry fields, which receive an average of 20 fungicide applications a year, compared with fewer than 10 applications in blueberry fields. Resistance frequencies as high as 90, 70, 50, and $40 \%$ to the QoI, SDHI, AP, and Hyd fungicides have been reported in Florida strawberry fields (Amiri et al. 2013, 2014). Similar resistance frequencies have also been reported in several other crops worldwide (Fernández-Ortuño et al. 2014; Leroux et al. 1999; Myresiotis et al. 2007). Fludioxonil is the sole fungicide to which actual field resistance has not been found in Florida (Amiri et al. 2013), whereas resistance has been sporadically reported in strawberry and blackberry in the eastern United States (Fernández-Ortuño et al. 2014; Li et al. 2014).

Fungicide resistance risk assessment, especially in "high-risk" pathogens such as $B$. cinerea, is crucial to enhance disease management. Although blueberry is ranked as the second most important berry crop in the United States, reports on fungicide resistance pertaining to $B$. cinerea and other pathogens from blueberry are very rare. In a recent study including 249 and $106 \mathrm{~B}$. cinerea isolates from California and Washington, respectively, resistance frequencies ranging between 20 and 66\% have been reported for the SDHI, AP, QoI, and Hyd fungicides (Saito et al. 2016). Overall, fungicide input in blueberry fields is lower compared with strawberry fields. However, Florida blueberry fields most likely receive more sprays than those in California and Washington because conducive weather conditions are more common and frequent in the southeastern United States. Consequently, higher resistance frequencies may be expected in Florida blueberry fields. Therefore, this study was conducted to (i) investigate the potential impact of population drift between strawberry and blueberry 
fields in central Florida on Botrytis occurrence in blueberry; (ii) determine the sensitivity levels in $B$. cinerea blueberry populations to fungicides from six registered chemical groups (i.e., PHT, QoI, SDHI, Hyd, AP, and PP); (iii) determine sensitivity levels to thiophanate-methyl and fluopyram, two fungicides that are not registered on blueberry but are on strawberry; and (iv) determine the occurrence of multiple fungicide resistance (MFR) phenotypes in commercial blueberry fields.

\section{Materials and Methods}

Fields surveyed. Twelve blueberry fields, planted to two SHB cultivars (Jewel or Emerald, predominant cultivars in Florida), were surveyed in 2014 and 2015. Six fields, grown at least 30 to $50 \mathrm{~km}$ distant from strawberry fields, were in Hillsborough $\left(28^{\circ} \mathrm{N}\right.$ parallel $)$, Alachua $\left(30^{\circ} \mathrm{N}\right.$ parallel), Orange (between the 28 and $29^{\circ} \mathrm{N}$ parallels), and Polk $\left(28^{\circ} \mathrm{N}\right.$ parallel) Counties (Table 1$)$. Six other blueberry fields planted within a few meters to up to $1 \mathrm{~km}$ from strawberry fields, were in Hillsborough, Citrus (south $29^{\circ} \mathrm{N}$ parallel) and Hernando (between the 28 and $29^{\circ} \mathrm{N}$ parallels) Counties (Table 1). The surveyed fields have been sprayed for more than a decade with several fungicides listed in Table 2, except thiophanate-methyl and fluopyram, in blueberry fields. In Florida, 90\% of strawberry fields are in Hillsborough County. Throughout this article, blueberry fields planted in the vicinity or not of strawberry fields are referred to as BB-Str(t) and BB-Str ${ }^{(-)}$, respectively.

Collection and storage of $B$. cinerea isolates. In total, 613 B. cinerea isolates were collected and tested in this study (Table 1). These included 308 and 305 isolates collected in 2014 and 2015, respectively, and 372 isolates from flowers and 241 isolates from fruit, respectively (Table 1). Surveyed fields were regularly sprayed with the fungicides included in this study (Table 2) since early 2000 for captan and most single-site fungicides, except for the SDHI penthiopyrad and fluopyram and the methyl benzimidazole carbamate (BMC) thiophanate-methyl, which are not registered on SHB cultivars. Fifty blueberry samples (blossoms and fruit) were collected from each field between February and May of each year, in separate bags to avoid cross-contaminations. Botrytis-like conidiophores visible on symptomatic samples were immediately transferred to agar media plates, as described below. Asymptomatic blossoms and fruit were surface disinfected in $0.05 \%$ sodium hypochlorite, rinsed in sterile distilled water, and incubated in saturated conditions to enhance sporulation. After 5 to 7 days incubation at $22^{\circ} \mathrm{C}$, samples were

Table 1. Geographic locations of blueberry fields surveyed and number of Botrytis isolates collected in this study

\begin{tabular}{|c|c|c|c|c|c|c|c|c|}
\hline \multirow[b]{3}{*}{ Field ${ }^{y}$} & \multirow[b]{3}{*}{ County } & \multirow[b]{3}{*}{ Strawberry ${ }^{z}$} & \multicolumn{6}{|c|}{ Number of isolates ${ }^{x}$} \\
\hline & & & \multicolumn{3}{|c|}{2014} & \multicolumn{3}{|c|}{2015} \\
\hline & & & $\sum$ & Flowers & Fruit & $\sum$ & Flowers & Fruit \\
\hline \multicolumn{9}{|l|}{ Not in vicinity } \\
\hline JSE & Hillsborough & BB-Str( ${ }^{(-)}$ & 14 & 9 & 5 & 10 & 8 & 2 \\
\hline DKC & Hillsborough & $\mathrm{BB}-\mathrm{Str}^{(-)}$ & 14 & 10 & 4 & 18 & 16 & 2 \\
\hline KLY & Hillsborough & $\mathrm{BB}-\mathrm{Str}^{(-)}$ & 11 & 2 & 9 & 16 & 4 & 12 \\
\hline HTF & Alachua & BB-Str ${ }^{(-)}$ & 15 & 10 & 5 & 30 & 29 & 1 \\
\hline BLS & Polk & BB-Str ${ }^{(-)}$ & 12 & 8 & 4 & 17 & 0 & 17 \\
\hline FZL & Orange & $\mathrm{BB}-\mathrm{Str}^{(-)}$ & 11 & 11 & 0 & 13 & 8 & 5 \\
\hline Total per year & $\ldots$ & $\ldots$ & 77 & 50 & 27 & 104 & 65 & 39 \\
\hline \multicolumn{9}{|l|}{ In vicinity } \\
\hline FCT & Citrus & BB-Str ${ }^{(+)^{*}}$ & 81 & 76 & 5 & 11 & 11 & 0 \\
\hline BRK & Hernando & BB-Str ${ }^{(+)^{*}}$ & 73 & 40 & 33 & 16 & 10 & 6 \\
\hline DME & Hillsborough & $\mathrm{BB}-\mathrm{Str}^{(+)}$ & 17 & 10 & 7 & 38 & 12 & 26 \\
\hline $\mathrm{JCL}$ & Hillsborough & BB-Str ${ }^{(+)}$ & 20 & 6 & 14 & 35 & 10 & 25 \\
\hline RYK & Hillsborough & BB-Str ${ }^{(+)}$ & 10 & 6 & 4 & $64^{*}$ & 28 & 36 \\
\hline TUM & Hillsborough & BB-Str ${ }^{(+)}$ & 30 & 21 & 9 & 37 & 27 & 10 \\
\hline Total per year & $\ldots$ & $\ldots$ & 231 & 159 & 72 & 201 & 98 & 103 \\
\hline Total general & $\ldots$ & $\ldots$ & 308 & 209 & 99 & 305 & 163 & 142 \\
\hline
\end{tabular}

$\times \sum$ indicates total number of Botrytis isolates collected from each field per year and field type.

${ }^{y}$ Letters are abbreviations of names for each blueberry field surveyed in this study in the vicinity of strawberry fields or not in the vicinity.

${ }^{\mathrm{z}} \mathrm{BB} \mathrm{Str}^{(-)}$indicates blueberry fields planted 30 to $50 \mathrm{~km}$ from strawberry fields and BB-Str ${ }^{(+)}$indicates blueberry fields planted in the vicinity of strawberry fields. In all, 50 flowers and fruit were collected from each field, except for those followed with an asterisk, from which 200 samples were collected.

Table 2. Characteristics, fungicide discriminatory doses, and media used to test for fungicide sensitivity in Botrytis cinerea

\begin{tabular}{|c|c|c|c|c|c|}
\hline Active ingredient (a.i.) ${ }^{w}$ & Trade name & a.i. $(\%)$ & Chemical group $^{x}$ & Test medium ${ }^{y}$ & Discriminatory doses $(\mu \mathrm{g} / \mathrm{ml})^{z}$ \\
\hline \multicolumn{6}{|l|}{$\overline{\text { Registered }}$} \\
\hline Pyraclostrobin (Pyra) & Cabrio & 20 & QoI & $1 \% \mathrm{MEA}$ & 10 \\
\hline Boscalid (Bosc) & Endura & 70 & SDHI & YBA & 5 \\
\hline Penthiopyrad (Penth) & Fontelis & 20.4 & SDHI & YBA & 3 \\
\hline Fenhexamid (Fenh) & Elevate & 50 & Hyd & $1 \%$ MEA & 1 and 50 \\
\hline Cyprodinil (Cypr) & Vangard & 75 & $\mathrm{AP}$ & $0.5 \% \mathrm{SA}$ & 1 and 25 \\
\hline Fludioxonil (Flud) & Medallion & 50 & $\mathrm{PP}$ & $1 \%$ MEA & 0.1 and 10 \\
\hline Captan (Capt) & Captec & 37.4 & PHT & $1 \% \mathrm{MEA}$ & 5 \\
\hline \multicolumn{6}{|l|}{ Nonregistered } \\
\hline Fluopyram (Flup) & Luna Privilege & 41.5 & SDHI & YBA & 2.5 \\
\hline Thiophanate-Methyl (ThM) & Topsin-M & 70 & $\mathrm{BMC}$ & $1 \% \mathrm{MEA}$ & 1 and 100 \\
\hline
\end{tabular}

${ }^{\mathrm{w}}$ Registered indicates fungicide registered in blueberry and strawberry and nonregistered indicates fungicides registered in strawberry only. Penthiopyrad is registered in lowbush blueberry only. Abbreviations in parentheses are used to refer to each fungicide throughout the article.

x QoI, SDHI, Hyd, AP, PP, PHT, and BMC indicate Quinone outside inhibitor, succinate dehydrogenase inhibitor, hydroxyanilide, anilino-pyrimidine, phenylpyrrole, phthalimide, and methyl benzimidazole carbamate fungicides, respectively.

y MEA, YBA, and SA indicate malt extract agar, yeast bacto acetate agar, and sucrose agar, respectively.

${ }^{\mathrm{z}}$ Discriminatory doses used to test fungicide sensitivity were based on previously determined doses or developed in this study. 
checked using a stereoscope and Botrytis-like conidiophores were transferred to general isolation medium containing $19.5 \mathrm{~g}$ of potato dextrose agar (PDA), $9.5 \mathrm{~g}$ of agar, $0.25 \mathrm{~g}$ of ampicillin, and $0.1 \mathrm{~g}$ of streptomycin in 1 liter of distilled water. Plates were incubated for $48 \mathrm{~h}$ at $22^{\circ} \mathrm{C}$ and a plug taken from the edge of a growing colony was transferred upside-down to a malt yeast extract agar (MYA) plate and incubated until profuse sporulation was observed; isolates were single spored and stored in $20 \%$ glycerol at $-80^{\circ} \mathrm{C}$.

Fungicides. Seven fungicides from six chemical groups registered for use in blueberry fields were used in this study - that is, pyraclostrobin (Cabrio EC; BASF Crop Protection, Research Triangle Park, NC), boscalid (Endura; BASF Crop Protection), fenhexamid (Elevate 50 WDG; Arysta Life Science, Cary, NC), cyprodinil (Vangard WG; Syngenta Crop Protection, Greensboro, NC), fludioxonil (Medallion WDG; Syngenta Crop Protection), penthiopyrad (Fontelis; Dupont Crop Protection, Wellington, DE), and the multisite fungicide captan (Captec 4L; Arysta LifeScience) (Table 2). Penthiopyrad is registered on lowbush blueberry but not on SHB. Additionally, two fungicides nonregistered on blueberry but registered on strawberry were tested: penthiopyrad, fluopyram SC 500 (Luna Privilege; Bayer CropScience, Research Triangle Park, NC), and thiophanate-methyl (Topsin-M 70WP; Cerexagri-Nisso LLC, King of Prussia, PA). Formulated products were dissolved in sterile distilled water to make $2,000 \mu \mathrm{g} / \mathrm{ml}$ stocks which were stored at $4^{\circ} \mathrm{C}$. Salicylhydroxamic acid (SHAM; Acros Organics, Fair Lawn, NJ) was added at $100 \mathrm{mg} /$ liter to malt extract agar (MEA) medium (Table 2) to inhibit alternative oxidase pathway when testing for sensitivity to pyraclostrobin (Amiri et al. 2010).

Fungicide sensitivity tests. Sensitivity to the aforementioned fungicides was evaluated based on a conidial germination assay described previously (Weber and Hahn 2011), with some modifications for the SDHI (Amiri et al. 2017). The discriminatory doses for cyprodinil, pyraclostrobin, fenhexamid, fludioxonil, and thiophanate-methyl were developed by Weber and Hahn (2011). For boscalid, the discriminatory dose of $5 \mu \mathrm{g} / \mathrm{ml}$ of was adjusted from Amiri et al. (2014) and Leroch et al. (2013), whereas the discriminatory doses for penthiopyrad and fluopyram were developed after comparing several $B$. cinerea isolates with different mutations in the SdhB subunit (Amiri et al. 2017). For captan, preliminary in vitro tests were conducted on a subsample of $40 \mathrm{~B}$. cinerea isolates using doses of $1.0,2.5$, and $5.0 \mu \mathrm{g} / \mathrm{ml}$. All isolates germinated and grew equally on captan at 1.0 and $2.5 \mu \mathrm{g} / \mathrm{ml}$, whereas some isolates were inhibited at $5.0 \mu \mathrm{g} / \mathrm{ml}$ and this dose was selected for further sensitivity tests.

The $613 \mathrm{~B}$. cinerea isolates were grown from $-80^{\circ} \mathrm{C}$ stock on MYA plates for 7 to 10 days or until profuse sporulation was observed. Conidia were washed in $10 \mathrm{ml}$ of sterile distilled water and filtered through cheesecloth, and concentration was determined using a

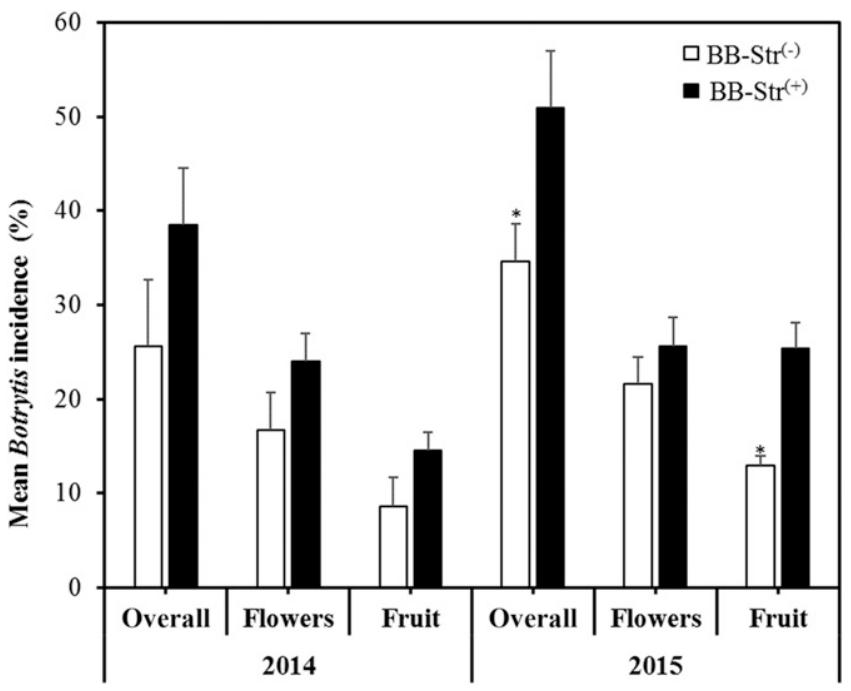

Fig. 1. Botrytis incidence on blueberry flowers and fruit collected from blueberry fields without (BB-Str ${ }^{(-)}$, open bars) or with (BB-Str ${ }^{(+)}$, filled bars) neighboring strawberry fields in 2014 and 2015. An asterisk on the vertical bars (standard deviations) indicates a significant difference at $P \leq 0.05$. hemacytometer and adjusted to $10^{6}$ conidia/ml. MEA (malt extract at $10 \mathrm{~g} /$ liter and agar at $15 \mathrm{~g} / \mathrm{liter}$ ), yeast acetate bacto agar (yeast extract $10 \mathrm{~g} /$ liter, bacto peptone at $10 \mathrm{~g} /$ liter, sodium acetate at $20 \mathrm{~g} /$ liter, and agar at $15 \mathrm{~g} /$ liter), and sucrose agar (sucrose at $5 \mathrm{~g} /$ liter and agar at $15 \mathrm{~g} /$ liter) were used to assess fungicide sensitivity (Table 2). Autoclaved molten $\left(55^{\circ} \mathrm{C}\right)$ media were amended with discriminatory doses of each fungicide (Table 2) and poured into 15-cm-diameter Petri dishes. Plates were placed on a self-adhesive transparent cellophane sheet $(12$ by $12 \mathrm{~cm}$ ), previously divided into 60 approximately 1-by1 -cm squares using a permanent marker. Two $7-\mu 1$ droplets of conidial suspension $\left(10^{6}\right.$ conidia/ml $)$ from each isolate were inoculated in each square, allowing testing of up to 60 isolates per plate. Plates containing respective media nonamended with fungicides were used as control. Germination was microscopically evaluated after 16 to $20 \mathrm{~h}$ incubation at $22^{\circ} \mathrm{C}$. Two replicate plates were used for each isolate and the test was repeated twice. Four different sensitivity phenotypes were distinguished based on the germination rate and germ tube length assessed microscopically using a scale of 0 to 4 , where $0=$ total inhibition, $1=$ low resistance ( 1 to 1.5 times the diameter of the conidium), $2=$ moderate resistance ( 2 to 4 times the diameter of the conidium), $3=$ highly resistant ( $>4$ times diameter of the conidium), and $4=$ germ tube length similar to the control plates. This scale was used to further determine resistance frequencies regardless of the resistance level well established in several reference publications.

In vivo sensitivity of $B$. cinerea to captan. Different sensitivity levels were observed among isolates tested in vitro on MEA amended with captan at $5 \mu \mathrm{g} / \mathrm{ml}$. To evaluate the efficacy of captan preventive applications, eight isolates (i.e., four isolates completely inhibited and four isolates that germinated in vitro) were selected for detachedfruit assays. Efficacy tests were conducted on detached blackberry fruit prepared and inoculated as described previously (Amiri et al. 2014). Blackberry fruit were used because blueberry fruit were not available all times and some preliminary tests on blueberry yielded a large number of contaminations. Test fruit were sprayed preventively with captan (Captec $4 \mathrm{~L}$ ) at 2.5 and $6.4 \mu \mathrm{l} / \mathrm{ml}$, corresponding to the lower and maximum label rates, respectively. Control fruit were sprayed with sterile water. Fruit were inoculated with $40 \mu$ l of a spore suspension of each isolate at $10^{5} \mathrm{spore} / \mathrm{ml}$. Eight replicate fruit were used for each isolate-fungicide combination and the experiment was done in duplicate and repeated twice. Fruit were incubated at $20^{\circ} \mathrm{C}$ for 5 days in saturated conditions and BFR incidence and severity were recorded.

Data analysis. Incidence of $B$. cinerea on blossoms collected from different commercial fields and BFR incidence on fruit at commercial maturity was estimated as the percentage of samples on which $B$. cinerea was detected. BFR incidence and severity on detached fruit to assess for efficacy of captan were expressed as percentage of fruit with BFR infection and reduction in diameter lesion compared with the control, respectively. All frequencies (\%) were logtransformed for analysis of variance (ANOVA) and least significant difference mean separation analyses at $P \leq 0.05$. For each fungicide, resistance frequencies were determined as percentage of isolates resistant (regardless of resistance level) among the total isolates from each field. Frequencies and mean resistance frequencies from BB-Str ${ }^{(+)}$ and BB-Str ${ }^{(-)}$fields (factors), each consisting of six replicate-fields, were subjected to an ANOVA and mean separation. All statistical analyses were performed using JMP software (JMP statistical software, version 12.1.0; SAS Institute Inc., Cary, NC).

\section{Results}

Incidence of $B$. cinerea on blueberry blossoms and fruit at commercial maturity. The incidence of $B$. cinerea ranged from 0 to $30 \%$ and from 0 to $58 \%$ in blueberry fields located far from any strawberry field $\left(\mathrm{BB}-\mathrm{Str}^{(-)}\right)$in 2014 and 2015, respectively, Respective frequency ranges in blueberry fields planted next to strawberry fields $\left(\mathrm{BB}-\mathrm{Str}^{(+)}\right.$) were 3 to $38 \%$ and 0 to $54 \%$ (data not shown). Mean B. cinerea incidence in the BB-Str ${ }^{(+)}$was $38.5 \%$, numerically higher than $35.7 \%$ in $\mathrm{BB}^{-\mathrm{Str}^{(-)}}$in 2014, whereas incidence was significantly higher $(51 \%)$ in $\mathrm{BB}_{-} \mathrm{Str}^{(+)}$in 2015 compared to $34.7 \%$ in the BB-Str ${ }^{(-)}$fields (Fig. 1). 
Fungicide resistance to six single-site fungicides. The overall resistance frequencies were numerically higher in $\mathrm{BB}-\mathrm{Str}^{(+)}$compared to BB-Str ${ }^{(-)}$fields for all fungicides tested. However, significant differences were observed for four and three out of six fungicides tested in 2014 and 2015, respectively (Fig. 2). Among the 77 B. cinerea isolates collected in 2014 from the BB-Str ${ }^{(-)}$fields, 5, 20, 22, 22, 43, and $96 \%$ were resistant to penthiopyrad, fenhexamid, cyprodinil, boscalid, pyraclostrobin, and thiophanate-methyl, respectively (Fig. 2A). The respective resistance frequencies were $14,59,41,62,91$, and $98 \%$, among the 231 isolates collected from the BB-Str ${ }^{(+)}$fields. In 2015, a similar trend was observed, except that resistance to fenhexamid was higher compared cyprodinil and boscalid than in 2014.

Resistance to thiophanate-methyl was the highest and was found in all fields at frequencies ranging between 76 and $100 \%$ regardless of the presence or not of strawberry fields in the vicinity of the blueberry field (Table 3). Resistance to pyraclostrobin was found in almost all fields and the frequency ranged between 0 and $100 \%$ in the BB-Str ${ }^{(-)}$fields versus 29.7 to $100 \%$ in the BB-Str ${ }^{(+)}$fields (Table 3). Fenhexamid and boscalid had the third highest resistance frequencies, which were significantly higher in the BB-Str ${ }^{(+)}$fields (Table 3; Fig. 2). Resistance to fenhexamid was two- and threefold higher in BB-Str ${ }^{(+)}$fields in 2014 and 2015, with average frequencies of 59 and $73 \%$, respectively, compared to 15.5 and $36.5 \%$, respectively, in BB-Str ${ }^{(-)}$fields. Similar trends and frequencies were observed for boscalid (Table 3). Resistance to cyprodinil was found in all BB-Str ${ }^{(+)}$fields but absent in two BB-Str ${ }^{(-)}$fields in both survey years and was significantly higher in BB-Str ${ }^{(+)}$fields in both years. Resistance to penthiopyrad was low and frequencies ranged from 0 to $10 \%$ in BB-Str ${ }^{(-)}$fields versus 0 to $43.8 \%$ in BB-Str ${ }^{(+)}$fields (Table 3; Fig. 2). Overall resistance frequency to fluopyram was 3 and $4 \%$ in 2014 and 2015, respectively, whereas $1.7 \%$ of isolates tested in 2015 had a reduced sensitivity to fludioxonil (data not shown).

Multiple-resistance fungicide phenotypes in blueberry fields. Only $0.6 \%$ of the 308 isolates collected in 2014 were sensitive to all fungicides, whereas none was sensitive among the 305 isolates collected in 2015 (data not shown). Isolates resistant to six fungicides (MFR6) simultaneously were predominant in 2014 in both BB-Str ${ }^{(-)}$ and BB-Str ${ }^{(+)}$fields, whereas isolates resistant to five fungicides (MFR5) were predominant in 2015 (Fig. 3). In addition to the MFR5 phenotype, more isolates were resistant to four fungicides (MFR4) at frequencies of 19 and 24\% in BB-Str ${ }^{(-)}$and BB-Str ${ }^{(+)}$ fields, respectively, in 2015. The MFR3 phenotype was found at relatively lower frequencies from 2 to $11 \%$, whereas the frequency of MFR2 isolates ranged from 5 to $18 \%$ (Fig. 3). Isolates resistant to one fungicide (MFR1) were found in all fields at frequencies of 3 to $16 \%$ but absent in the BB-Str ${ }^{(+)}$fields in 2015 .

Sensitivity phenotypes to the multisite captan. Three discriminatory doses of captan $(1,2.5$, and $5 \mu \mathrm{g} / \mathrm{ml})$ were tested initially on a subsample of 40 Botrytis isolates. Those tested on 1.0 or $2.5 \mu \mathrm{g} / \mathrm{ml}$ germinated and had a germ tube length similar to the nonamended MEA control plates (data not shown). However, at $5 \mu \mathrm{g} / \mathrm{ml}$, germination and germ tube length were completely or significantly inhibited in a group of isolates (captan sensitive [Capt $\left.{ }^{\mathrm{S}}\right]$ ), whereas another group of isolates had a germ tube length similar to control plates (captan reduced sensitivity [Capt $\left.{ }^{\mathrm{RS}}\right]$ ). In 2014, the overall frequency of $\mathrm{Capt}^{\mathrm{S}}$ and $\mathrm{Capt}^{\mathrm{RS}}$ was 58.2 and $41.8 \%$, respectively (Fig. 4). The respective frequencies were 52.9 and $47.1 \%$ in 2015 . The frequency of $\mathrm{Capt}^{\mathrm{RS}}$ isolates was equally distributed in BB-Str ${ }^{(-)}$and BB-Str ${ }^{(+)}$(data not shown).

The lower label rate of captan did not control the Capt ${ }^{\mathrm{RS}}$ isolates after 5 days at $20^{\circ} \mathrm{C}$ on detached blackberry fruit and reduced incidence and severity of $\mathrm{Capt}^{\mathrm{S}}$ by $50 \%$ (Table 4). The highest label rate of captan fully controlled gray mold on fruit inoculated with Capt ${ }^{\mathrm{S}}$ and significantly reduced incidence and severity of $\mathrm{Capt}^{\mathrm{RS}}$ isolates for up to 5 days compared with the lower label rate (Table 4).

\section{Discussion}

Considered together, results from this study indicate that SHB cultivars may be at moderate risk for blossom blight infections depending on weather conditions occurring during the bloom period. Flowers of SHB cultivars Jewel and Emerald, the two main cultivars grown in Florida, are more susceptible to Botrytis infections than commercially ripe fruit because incidence was twice as high on flowers as on fruit. Previous studies reported similar findings on other SHB cultivars such as Gulfcoast and Cooper (Smith 1998), rabbiteye cultivars, and northern highbush blueberry cultivars (Bensch and Guerrero 2001; Rivera et al. 2013; Smith 1998). Generally, SHB cultivars are considered less prone to Botrytis infections than lowbush cultivars (Williamson et al. 2015). However, a study comparing SHB to rabbiteye cultivars reported that flowers of the SHB cultivar Gulfcoast were significantly more susceptible to Botrytis infections than many other rabbiteye cultivars 4 weeks under wet conditions (Smith 1998). The phenological stage can have an impact on Botrytis occurrence, as shown by Smith (1998), who found that all flowers of Gulfcoast were equally susceptible to infections at stages 2 to 7 , whereas flowers of the rabbiteye cultivars Tifblue, Premier, or Climax were more susceptible to infections at stages 5 to 7 (full bloom). In another study on northern highbush blueberry in Chile, the recovery of Botrytis spp. was significantly lower at petal fall compared with late pink and full bloom stages (Rivera et al. 2013). In our study, sampling of

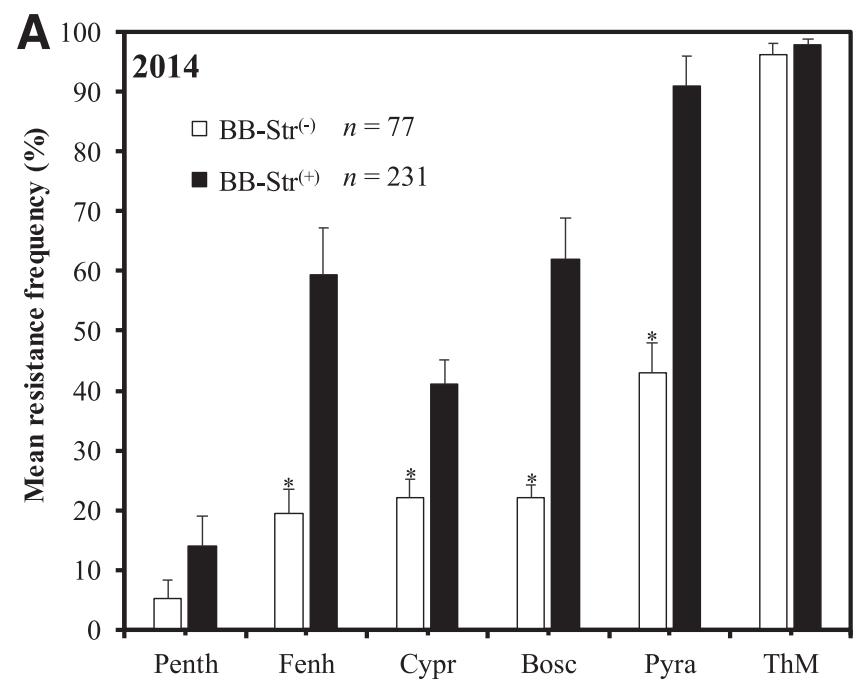

B

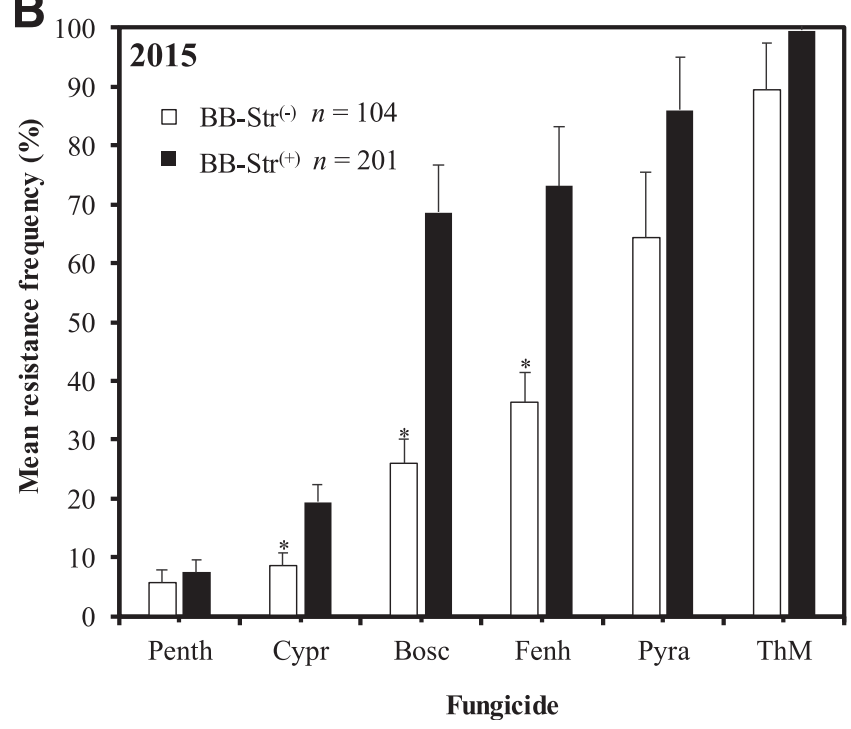

Fig. 2. Overall resistance frequencies to six fungicides in Botrytis cinerea isolates from blueberry fields without (BB-Str ${ }^{(-)}$, open bars) or with (BB-Str ${ }^{(+)}$, filled bars) neighboring strawberry fields in A, 2014 and B, 2015. An asterisk on the vertical bars (standard deviations) indicates significant differences at $P \leq 0.05$. Pyra, Bosc, Penth, Fenh, Cypr, and ThM refer to pyraclostrobin, boscalid, penthiopyrad, fenhexamid, cyprodinil, and thiophanate-methyl, respectively. 
most of the flowers was conducted at full bloom stage (5 to 7) and, therefore, our results should reflect infections events occurring in commercial blueberry in central Florida at this particular phenological stage.

Flowers and fruit collected from blueberry fields planted next to strawberry fields $\left(\mathrm{BB}-\mathrm{Str}^{(+)}\right)$showed increased $B$. cinerea incidence compared with blueberry fields distant from any strawberry field $\left(\mathrm{BB}-\mathrm{Str}^{(-)}\right)$in central Florida. Dispersal of Botrytis conidia has been reported to occur through wind, water splash, and insects (Holtz et al. 2009; Xu et al. 2012) and was found to occur from $0.6 \mathrm{~m}$ to a dozen meters from the source, depending on the wind velocity and host canopy (Chastagner et al. 1978; Holtz et al. 2009; Johnson and Powelson 1983; Thomas et al. 1988). In tomato fields in California, conidia of Botrytis were found $8 \mathrm{~m}$ from the inoculum source at a wind velocity of $0.5 \mathrm{~km} / \mathrm{h}$ (Chastagner et al. 1978), and the tomato canopy was suggested to play a role in retaining a large amount of inoculum within it and preventing conidia from long-distance spread (Chastagner et al. 1978). The BB-Str ${ }^{(+)}$surveyed in this study were less than $15 \mathrm{~m}$ from strawberry fields and the wind velocity in the flat Tampa Bay area, where most of the BB-Str ${ }^{(+)}$are located, is significantly faster than $0.5 \mathrm{~km} / \mathrm{h}$ because of continuous air currents from the Gulf. It can be speculated that the canopy of strawberry is less limiting for conidial dispersal than the tomato canopy which, therefore, may explain the increased infection rates in $\mathrm{BB}-\mathrm{Str}^{(+)}$fields. Chastagner et al. (1978) showed that the incidence of tomato plants killed by Botrytis spp. decreased with increasing distance from the inoculum source, except when a new tomato field is planted next to a field with profuse Botrytis sporulation. The highest peak of Botrytis inoculum commonly occurring in strawberry fields from February to May, when abundant numbers of receptive blueberry flowers are present in fields, most likely intensifies fungal population drifts within such short distances. Further studies using molecular markers are needed to investigate populations and gene flow between fields because this has a considerable impact on fungicide resistance development and efficacy of current management strategies.

Because of the considerably reduced fungicide input compared with strawberry fields, the resistance frequencies in blueberry fields were higher than expected. Overall, the resistance frequencies observed in Florida blueberry fields were very similar to those reported in strawberry (Amiri et al. 2013, 2014; Fernández-Ortuño et al. 2014; Leroch et al. 2013), a crop that receives twice the number of blueberry sprays. Although not registered for use on blueberry, resistance to the BMC thiophanate-methyl was particularly high $(>90 \%)$ regardless of the closeness to strawberry fields. This is most likely due to cross-resistance with benomyl, which was registered but discontinued on blueberry and for which resistance in Botrytis spp. has been widely reported (LaMondia and Douglas 1997; Lennox and
Spotts 2003; Northover and Matteoni 1986; Pepin and MacPherson 1982; Tanović and Ivanović 2010). Two decades ago, benomyl failed to reduce Botrytis blossom blight on blueberry cultivars Climax and Gulfcoast but resistance was not investigated (Smith 1998). This reinforces the general idea that resistance to certain fungicides is highly persistent even after being discontinued for a long period. Resistance to the QoI fungicide pyraclostrobin was 40 to $90 \%$ but was lower in BB-Str ${ }^{(-)}$compared with BB-Str ${ }^{(+)}$fields. In addition to being used as a solo product in Cabrio EG, pyraclostrobin is premixed with the SDHI boscalid as Pristine and has been used in blueberry fields since 2005 . The SDHI boscalid showed the third and fourth highest resistance frequency in 2014 and 2015, respectively. Interestingly, resistance frequencies to boscalid were lower than those reported in Florida strawberry fields, where more than $80 \%$ of isolates were boscalid resistant (Amiri et al. 2013, 2014). Herein, the presence of a strawberry field in the vicinity of the blueberry field resulted in significantly higher resistance frequencies to this SDHI, which suggest that blueberry fields are at a higher risk when planted next to hosts highly susceptible to Botrytis spp. because they may serve as a sink for resistant populations spreading from neighboring fields. Although penthiopyrad is registered on lowbush blueberry only and fluopyram is not registered, low resistance frequencies of

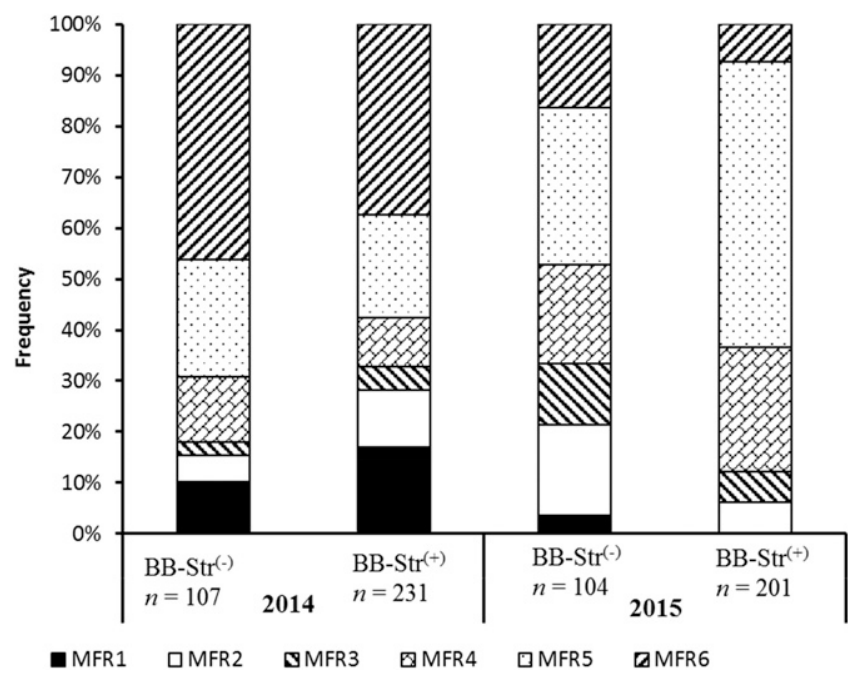

Fig. 3. Overall frequency of multiple fungicide resistance (MFR) phenotypes in Botrytis cinerea isolates from blueberry fields without $\left(\mathrm{BB}-\mathrm{Str}^{(-)}\right)$or with $\left(\mathrm{BB}-\mathrm{Str}^{(+)}\right)$neighboring strawberry fields in 2014 and 2015. MFR1, MFR2, MFR3, MFR4, MFR5, and MFR6 indicate isolates resistant to one fungicide or, simultaneously, resistant to two, three, four, five, and six fungicides, respectively (Amiri et al. 2013).

Table 3. Frequency of resistance to six fungicides in Botrytis cinerea isolates collected from 12 blueberry fields in Florida

\begin{tabular}{|c|c|c|c|c|c|c|c|c|c|c|c|c|c|c|c|c|}
\hline \multirow[b]{3}{*}{ Field } & \multirow[b]{3}{*}{ County } & \multirow[b]{3}{*}{ Strawberry $^{\mathbf{z}}$} & \multicolumn{14}{|c|}{ Resistance frequency $(\%)^{\mathrm{y}}$} \\
\hline & & & \multicolumn{7}{|c|}{2014} & \multicolumn{7}{|c|}{2015} \\
\hline & & & $n$ & Pyra & Bosc & Penth & Fenh & Cypr & ThM & $\bar{n}$ & Pyra & Bosc & Penth & Fenh & Cypr & ThM \\
\hline JSE & Hillsborough & BB-Str ${ }^{(-)}$ & 14 & 78.6 & 42.9 & 7.1 & 42.9 & 35.7 & 92.9 & 10 & 80.0 & 10.0 & 0.0 & 50.0 & 0.0 & 100 \\
\hline DKC & Hillsborough & BB-Str ${ }^{(-)}$ & 14 & 71.4 & 35.7 & 7.1 & 35.7 & 28.6 & 92.9 & 18 & 100.0 & 33.3 & 5.6 & 44.4 & 5.6 & 100 \\
\hline KLY & Hillsborough & BB-Str ${ }^{(-)}$ & 11 & 27.3 & 18.2 & 9.1 & 0.0 & 0.0 & 100.0 & 16 & 81.3 & 37.5 & 6.3 & 31.3 & 12.5 & 100 \\
\hline HTF & Allachua & BB-Str ${ }^{(-)}$ & 15 & 33.3 & 0.0 & 0.0 & 13.3 & 33.3 & 100.0 & 30 & 50.0 & 36.7 & 10.0 & 36.7 & 13.3 & 77 \\
\hline BLS & Polk & BB-Str ${ }^{(-)}$ & 12 & 0.0 & 0.0 & 0.0 & 0.0 & 0.0 & 100.0 & 17 & 52.9 & 0.0 & 0.0 & 35.3 & 0.0 & 76 \\
\hline FZL & Orange & BB-Str ${ }^{(-)}$ & 11 & 36.4 & 36.4 & 9.1 & 18.2 & 27.3 & 90.9 & 13 & 30.8 & 23.1 & 7.7 & 23.1 & 15.4 & 100 \\
\hline FCT & Citrus & BB-Str ${ }^{(+)}$ & 81 & 91.4 & 69.1 & 13.6 & 61.7 & 44.4 & 95.1 & 11 & 100.0 & 81.8 & 0.0 & 90.9 & 27.3 & 100 \\
\hline BRK & Hernando & BB-Str( ${ }^{(+)}$ & 73 & 93.2 & 69.9 & 19.2 & 54.8 & 43.8 & 100.0 & 16 & 100.0 & 93.8 & 43.8 & 56.3 & 18.8 & 100 \\
\hline DME & Hillsborough & BB-Str ${ }^{(+)}$ & 17 & 88.2 & 64.7 & 0.0 & 64.7 & 23.5 & 100.0 & 38 & 94.7 & 86.8 & 0.0 & 86.8 & 18.4 & 97 \\
\hline JCL & Hillsborough & BB-Str ${ }^{(+)}$ & 20 & 100.0 & 55.0 & 10.0 & 75.0 & 25.0 & 100.0 & 35 & 100.0 & 37.1 & 0.0 & 88.6 & 37.1 & 100 \\
\hline RYK & Hillsborough & BB-Str( ${ }^{(+)}$ & 10 & 90.0 & 50.0 & 0.0 & 90.0 & 30.0 & 90.0 & 64 & 100.0 & 92.2 & 6.3 & 89.1 & 12.5 & 100 \\
\hline TUM & Hillsborough & BB-Str ${ }^{(+)}$ & 30 & 80.0 & 30.0 & 16.7 & 40.0 & 50.0 & 100.0 & 37 & 29.7 & 24.3 & 10.8 & 18.9 & 13.5 & 100 \\
\hline
\end{tabular}

${ }^{\mathrm{y}}$ Resistance frequency in percentage relative to the total number of isolates tested from each field. $n=$ number of isolates tested for each field and specific year. Pyra, Bosc, Penth, Fenh, Cypr, and ThM refer to pyraclostrobin, boscalid, penthiopyrad, fenhexamid, cyprodinil, and thiophanate-methyl, respectively. ${ }^{2} \mathrm{BB}_{-\mathrm{Str}^{(-)}}$and BB-Str ${ }^{(+)}$indicate blueberry fields planted far from $(30$ to $50 \mathrm{~km})$ and in the vicinity of strawberry fields, respectively. 
20 and $4 \%$, respectively, were found in blueberry fields. This is likely due to cross-resistance with boscalid in selected field mutants carrying the mutations $\mathrm{H} 272 \mathrm{Y}$, N230I, or P225F that are known to confer resistance to three SDHI (Amiri and Peres 2014; Leroux et al. 2010), whereas the H272R mutants, predominant in Florida (Amiri and Peres 2014), are only resistant to boscalid. The relatively low resistance frequency to both SDHI in strawberry fields (Amiri et al. 2014) may explain that differences between BB-Str ${ }^{(-)}$ and BB-Str ${ }^{(+)}$fields were not significant contrary to the other SDHI boscalid.

The Hyd fenhexamid has been used in blueberry fields either as a solo product (Elevate $50 \mathrm{WG}$ ) or mixed with captan (Captivate). The resistance frequencies seen in BB-Str ${ }^{(-)}$fields ranged between 20 and $40 \%$, significantly lower compared with BB-Str ${ }^{(+)}$fields where resistance frequencies as high as $70 \%$ were found. The levels of resistance observed in the BB-Str ${ }^{(-)}$fields are similar to those reported $(29 \%)$ in blueberry fields from California and Washington State (Saito et al. 2016), whereas those in BB-Str ${ }^{(+)}$were similar or slightly higher than

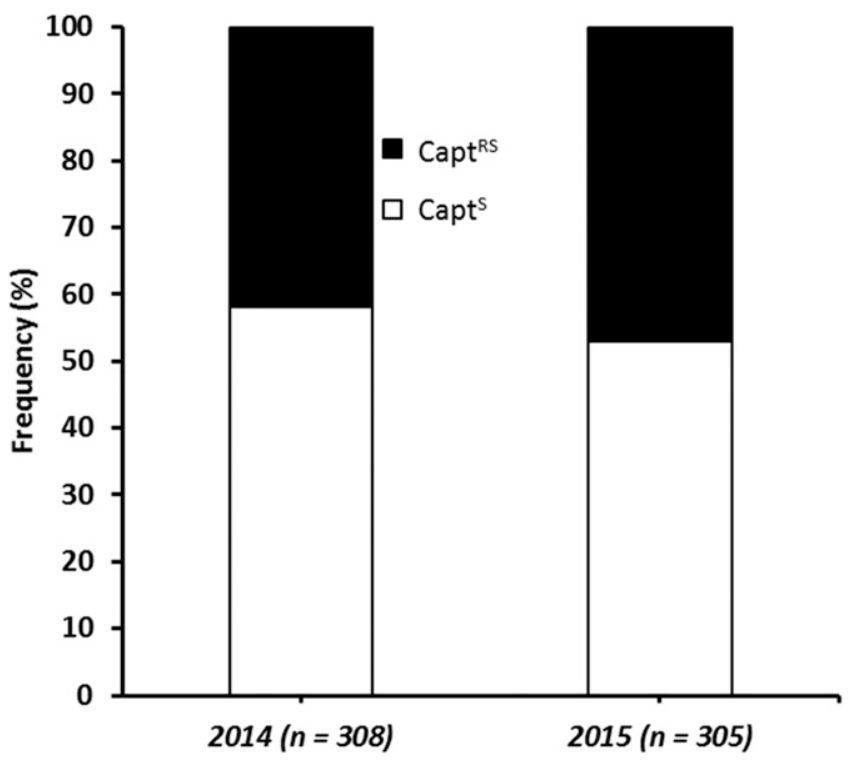

Fig. 4. Overall frequency of sensitivity phenotypes in Botrytis cinerea isolates from blueberry to the multisite captan in 2014 and 2015. Capts ${ }^{\text {}}$ (open bars) and Capt ${ }^{R S}$ (filled bars) indicate isolates sensitive and with reduced sensitivity to captan in vitro, respectively; $n$ indicates the total number of isolates tested each year. frequencies reported in Florida strawberry fields (Amiri and Peres 2014; Amiri et al. 2013; Fernández-Ortuño et al. 2014). Resistance to cyprodinil was relatively low, ranging from 10 to $20 \%$ in BB$\mathrm{Str}^{(-)}$and from 20 to $30 \%$ in BB-Str ${ }^{(+)}$fields, similar to recent frequencies reported in California (20\%) and Washington state (29\%) (Saito et al. 2016) but lower than those reported in Florida (50\%) strawberry fields (Amiri et al. 2013). This is likely due to the extensive use of pyrimethanil in strawberry in addition to cyprodinil premixed with fludioxonil (Switch 62.5 WG), whereas only the later product has been used in blueberry fields since 2005. Fludioxonil is the only fungicide for which actual resistance was not found in blueberry fields and only $1.7 \%$ of the isolates tested showed reduced sensitivity significantly lower than the $70 \%$ reported in Washington State (Saito et al. 2016). These isolates are controlled by the full label rate of fludioxonil (Amiri et al. 2013) but the existence of such populations warrants cautious use of Switch to avoid selecting for actual resistant isolates.

Multisite fungicides are a useful tool recommended for rotations or tank mixtures with single-site fungicides to improve efficacy and curb resistance development. However, resistance to some multisite fungicides (i.e., captan) in $B$. cinerea has been reported previously in strawberry, raspberry, grape, and bean (Barak and Edgington 1984; Pepin and MacPherson 1982). Overall, 40 to $47 \%$ of blueberry isolates from Florida were found to have a reduced sensitivity to cap$\tan$. These frequencies are higher than those found in strawberry (28\%) and slightly lower than those reported on raspberry (54 to $66 \%$ ) (Pepin and MacPherson 1982). The discriminatory dose of cap$\tan (5 \mu \mathrm{g} / \mathrm{ml})$ used to screen for sensitivity on MEA after preliminary tests is significantly lower than the $100 \mu \mathrm{g} / \mathrm{ml}$ used by Pepin and MacPherson (1982) on PDA, a rich medium not suitable for fungicide sensitivity testing, especially in Botrytis spp. (Weber and Hahn 2011). Interestingly, resistance to captan was found to be age dependent because spores from 3-week-old cultures lost their resistance but the resistance phenotype was regained 1 week after exposure to captan (Barak and Edgington 1984). In Florida strawberry and blueberry fields, weekly sprays of captan are very common throughout the growing season, even when disease pressure is low (Legard et al. 2005), which can continuously select for captan-resistant isolates. Although not investigated in the current study, it is likely that some resistance or reduced sensitivity to captan occurs in strawberry fields as well. Consequently, sprays of captan should be alternated with other multi- and single-site fungicides having different modes of action. Findings from the detached-fruit assay indicate that the highest label rate of captan should be recommended to improve efficacy and reduce selection of captan-reduced sensitivity.

Table 4. Gray mold incidence and severity on blackberry fruit treated preventively with two rates of captan and inoculated with Botrytis cinerea isolates with different in vitro sensitivity phenotypes

\begin{tabular}{|c|c|c|c|c|c|}
\hline \multirow[b]{3}{*}{ Isolate } & \multirow[b]{3}{*}{ Phenotype $^{\mathrm{z}}$} & \multicolumn{4}{|c|}{ Gray mold incidence and severity on fruit treated with captan aty } \\
\hline & & \multicolumn{2}{|c|}{$2.5 \mu 1 / \mathrm{ml}$ (low rate) } & \multicolumn{2}{|c|}{$6.4 \mu 1 / \mathrm{ml}$ (high rate) } \\
\hline & & Incidence & Severity & Incidence & Severity \\
\hline \multicolumn{6}{|l|}{ Sensitive } \\
\hline Bc13-411 & $\mathrm{Capt}^{\mathrm{S}}$ & $58.3 \mathrm{~b}$ & $52.3 \mathrm{~b}$ & $0.0 \mathrm{c}$ & $0.0 \mathrm{~b}$ \\
\hline Bc13-412 & $\mathrm{Capt}^{\mathrm{S}}$ & $50.0 \mathrm{~b}$ & $47.8 \mathrm{~b}$ & $0.0 \mathrm{c}$ & $0.0 \mathrm{~b}$ \\
\hline Bc13-414 & $\mathrm{Capt}^{\mathrm{S}}$ & $54.2 \mathrm{~b}$ & $46.7 \mathrm{~b}$ & $0.0 \mathrm{c}$ & $0.0 \mathrm{~b}$ \\
\hline Bc13-423 & $\mathrm{Capt}^{\mathrm{S}}$ & $45.8 \mathrm{~b}$ & $47.3 \mathrm{~b}$ & $0.0 \mathrm{c}$ & $0.0 \mathrm{~b}$ \\
\hline Mean & $\ldots$ & 52.1 & 48.5 & 0.0 & 0.0 \\
\hline \multicolumn{6}{|c|}{ Reduced sensitivity } \\
\hline Bc13-404 & $\mathrm{Capt}^{\mathrm{RS}}$ & $100.0 \mathrm{a}$ & $80.2 \mathrm{a}$ & $25.0 \mathrm{a}$ & $28.5 \mathrm{a}$ \\
\hline Bc13-410 & $\mathrm{Capt}^{\mathrm{RS}}$ & $100.0 \mathrm{a}$ & $75.5 \mathrm{a}$ & $20.8 \mathrm{ab}$ & $25.8 \mathrm{a}$ \\
\hline Bc13-438 & $\mathrm{Capt}^{\mathrm{RS}}$ & $100.0 \mathrm{a}$ & $70.9 \mathrm{a}$ & $29.2 \mathrm{a}$ & $33.5 \mathrm{a}$ \\
\hline Bc13-441 & $\mathrm{Capt}^{\mathrm{RS}}$ & $100.0 \mathrm{a}$ & $81.2 \mathrm{a}$ & $16.7 \mathrm{~b}$ & $25.7 \mathrm{a}$ \\
\hline Mean & $\ldots$ & 100.0 & 77.0 & 22.9 & 28.4 \\
\hline
\end{tabular}


The occurrence of a large number of multifungicide-resistant (MFR) isolates and the presence of hosts highly susceptible to Botrytis infections in the vicinity may render blossom blight and BFR management more challenging in blueberry fields. Continuous monitoring of resistance and further investigation of population flow and the role of multisite fungicides in current spray programs are needed to curb resistance development and develop more suitable management strategies in blueberry fields.

\section{Acknowledgments}

We thank P. Harmon, University of Florida, for assisting with sampling; and E. and R. Martin, R. Onofre, and R. Quijia, Gulf Coast Research and Education Center, for technical assistance.

\section{Literature Cited}

Amiri, A., Brannen, P. M., and Schnabel, G. 2010. Reduced sensitivity in Monilinia fructicola field isolates from South Carolina and Georgia to respiration inhibitor fungicides. Plant Dis. 94:737-743.

Amiri, A., Heath, S. M., and Peres, N. A. 2013. Phenotypic characterization of multifungicide resistance in Botrytis cinerea isolates from strawberry fields in Florida. Plant Dis. 97:393-401.

Amiri, A., Heath, S. M., and Peres, N. A. 2014. Resistance to fluopyram, fluxapyroxad, and penthiopyrad in Botrytis cinerea from strawberry. Plant Dis. 98:532-539.

Amiri, A., Mulvaney, K. A., and Pandit, L. K. 2017. First Report of Resistance to Fluxapyroxad and Fluopyram in Botrytis cinerea From Commercial Apple Orchards in Washington State. Plant Dis. 101:508.

Amiri, A., and Peres, N. A. 2014. Diversity in the erg27 gene of Botrytis cinerea field isolates from strawberry defines different levels of resistance to the hydroxyanilide fenhexamid. Plant Dis. 98:1131-1137.

Barak, E., and Edgington, I. V. 1984. Botrytis cinerea resistant to captan: The effect of inoculum age and type on response to the fungicide. Can. J. Plant Pathol. 6:211-214.

Batra, L. R. 1983. Monilinia vaccinii-corymbosi (Sclerotiniaceae): Its biology on blueberry and comparison with related species. Mycologia 75:131-152.

Bensch, E., and Guerrero, J. 2001. Eficacia de benomilo+captan y BC1000 en el control de Botrytis cinerea y Alternaria alternata en arándano alto (Vaccinium corymbosum) cv. Bluejay. Agro Sur 29:12-19.

Burchhardt, K. M., and Cubeta, M. A. 2015. Population structure of the blueberry pathogen Monilinia vaccinii-corymbosi in the United States. Phytopathology 105:533-541.

Chastagner, G. A., Ogawa, J. M., and Manji, B. T. 1978. Dispersal of conidia of Botrytis cinerea in tomato fields. Phytopathology 68:1172-1176.

Fernández-Ortuño, D., Grabke, A., Bryson, P. K., Amiri, A., Peres, N. A., and Schnabel, G. 2014. Fungicide resistance profiles in Botrytis cinerea from strawberry fields in seven southern U.S. States. Plant Dis. 98:825-833.

Gillett, J. M., and Schilder, A. C. 2009. Environmental requirements for infection of blueberry Fruit by Colletotrichum acutatum. Acta Hortic. 810:355-360.

Holtz, G., Coertze, S., and Williamson, B. 2009. The ecology of Botrytis on plant surfaces. Pages 9-27 in: Botrytis: Biology, Pathology and Control. Y. Elad, B. Williamson, P. Tudzynski, and N. Delen, eds. Kluwer Academic Publishers, Dordrecht, The Netherlands.

Johnson, K. B., and Powelson, M. L. 1983. Analysis of spore dispersal gradients of Botrytis cinerea and gray mold disease gradients in snap beans. Phytopathology 73:741-746.
LaMondia, J. A., and Douglas, S. M. 1997. Sensitivity of Botrytis cinerea from Connecticut green houses to benzimidazole and dicarboxamide fungicides. Plant Dis. 81:729-732.

Legard, D. E., MacKenzie, S. J., Mertely, J. C., Chandler, C. K., and Peres, N. A. 2005. Development of a reduced use fungicide program for control of Botrytis fruit rot on annual winter strawberry. Plant Dis. 89:1353-1358.

Lennox, C. L., and Spotts, R. A. 2003. Sensitivity of Botrytis cinerea populations from pear-related sources to benzimidazole and dicarboxamide fungicides. Plant Dis. 87:645-649.

Leroch, M., Plesken, C., Weber, R. W. S., Kauff, F., Scalliet, G., and Hahn, M. 2013. Gray mould populations in German strawberry fields are resistant to multiple fungicides and dominated by a novel clade closely related to Botrytis cinerea. Appl. Environ. Microbiol. 79:159-167.

Leroux, P., Chapeland, F., Desbrosses, D., and Gredt, M. 1999. Patterns of crossresistance to fungicides in Botryotinia fuckeliana (Botrytis cinerea) isolates from French vineyards. Crop Prot. 18:687-697.

Leroux, P., Gredt, M., Leroch, M., and Walker, A. S. 2010. Exploring mechanisms of resistance to respiratory inhibitors in field strains of Botrytis cinerea, the causal agent of gray mold. Appl. Environ. Microbiol. 76:6615-6630.

Li, X., Fernández-Ortuño, D., Grabke, A., and Schnabel, G. 2014. Resistance to fludioxonil in Botrytis cinerea isolates from blackberry and strawberry. Phytopathology 104:724-732.

Myresiotis, C. K., Karaoglanidis, G. S., and Tzavella-Klonari, K. 2007. Resistance of Botrytis cinerea isolates from vegetable crops to anilinopyrimidine, phenylpyrrole, hydroxyanilide, benzimidazole, and dicarboximide fungicides. Plant Dis. 91: 407-413.

Northover, J., and Matteoni, J. A. 1986. Resistance of Botrytis cinerea to benomyl and iprodione in vineyards and greenhouses after exposure to the fungicides alone or mixed with captan. Plant Dis. 70:398-402.

Pepin, H. S., and MacPherson, E. A. 1982. Strains of Botrytis cinerea resistant to benomyl and captan in the field. Plant Dis. 66:404-405.

Rivera, S. A., Zoffoli, J. P., and Latorre, B. A. 2013. Infection risk and critical period for postharvest control of gray mold (Botrytis cinerea) on blueberry in Chile. Plant Dis. 97:1069-1074.

Saito, S., Michailides, T. J., and Xiao, C. L. 2016. Fungicide resistance profiling in Botrytis cinerea populations from blueberry in California and Washington State and their impact on control of gray mold. Plant Dis. 100: 2087-2093.

Smith, J. B. 1998. Botrytis blossom blight of southern blueberries: Cultivar susceptibility and effect of chemical treatments. Plant Dis. 82:924-927.

Tanović, B., and Ivanović, M. 2010. First report of benomyl resistance in Botrytis cinerea isolates on raspberry in Serbia. Plant Dis. 94:486.

Thomas, C. S., Marois, J. J., and English, J. T. 1988. The effect of wind speed, temperature, and relative humidity on development of aerial mycelium and conidia of Botrytis cinerea on grape. Phytopathology 78:260-265.

USDA-NASS. 2016. Florida Agricultural Overview. Online publication. United States Department of Agriculture-National Agricultural Statistics Service. https://quickstats.nass.usda.gov/

Weber, R. W. S., and Hahn, M. 2011. A rapid and simple method for determining fungicide resistance in Botrytis. J. Plant Dis. Prot. 118:17-25.

Williamson, J. G., Olmstead, J. W., and Lyrene, P. M. 2015. Florida's commercia blueberry industry. Online publication. Horticultural Sciences Department, UFIFAS. http://edis.ifas.ufl.edu/ac031

Xu, X., Wedgwood, E., Berrie, A. M., Allen, J., and O'Neill, T. M. 2012. Management of raspberry and strawberry grey mould in open field and under protection. A review. Agron. Sustain. Dev. 32:531-543. 